\begin{tabular}{|c|c|}
\hline Title & $\begin{array}{l}\text { A nalytical solutions describing the phase separation driven by a free energy functional containing a long-range } \\
\text { interaction term }\end{array}$ \\
\hline Author(s) & Ohnishi, I samu; Nishiura, Y asumasa; Imai, Masaki; Matsushita, Y ushu \\
\hline Citation & $\begin{array}{l}\text { Chaos: A n Interdisciplinary Journal of Nonlinear Science, 9(2), 329-341 } \\
\text { https://doi.org/10.1063/1.166410 }\end{array}$ \\
\hline Issue Date & 1999-06 \\
\hline Doc URL & http:/hdl.handle.net/2115/39895 \\
\hline Rights & $\begin{array}{l}\text { Copyright } 1999 \text { A merican Institute of Phy sics. This article may be downloaded for personal use only. A ny other use } \\
\text { requires prior permission of the author and the A merican Institute of Physics. The following article appeared in Chaos, } \\
\text { vol.9, pp.329-341, } 1999 \text { and may be found at https://dx.doi.org/10.1063/1.166410 }\end{array}$ \\
\hline Type & article \\
\hline File Information & nishiura 60.pdf \\
\hline
\end{tabular}

Instructions for use 


\title{
Analytical solutions describing the phase separation driven by a free energy functional containing a long-range interaction term
}

\author{
Isamu Ohnishia) \\ Department of Computer Sciences and Information Mathematics, Faculty of Electro-Communications, \\ The University of Electro-Communications, Chofu, Tokyo, 182, Japan \\ Yasumasa Nishiura \\ Laboratory of Nonlinear Studies and Computations, Research Institute for Electronic Science, \\ Hokkaido University, Kita-ku, Sapporo, 060, Japan \\ Masaki Imai \\ Department of Computer Sciences and Information Mathematics, Faculty of Electro-Communications, \\ The University of Electro-Communications, Chofu, Tokyo, 182, Japan \\ Yushu Matsushita \\ Neutron Scattering Laboratory, The Institute for Solid State Physics, University of Tokyo, Tokai, Naka-gun, \\ Ibaraki, 319-11, Japan
}

(Received 6 July 1998; accepted for publication 6 March 1999)

\begin{abstract}
We are primarily concerned with the variational problem with long-range interaction. This functional represents the Gibbs free energy of the microphase separation of diblock copolymer melts. The critical points of this variational problem can be regarded as the thermodynamic equilibrium state of the phase separation phenomenon. Experimentally it is well-known in the diblock copolymer problem that the final equilibrium state prefers periodic structures such as lamellar, column, spherical, double-diamond geometries and so on. We are interested in the characterization of the periodic structure of the global minimizer of the functional (corresponding to the strong segregation limit). In this paper we completely determine the principal part of the asymptotic expansion of the period with respect to $\varepsilon$ (interfacial thickness), namely, we estimate the higher order error term of the period with respect to $\varepsilon$ in a mathematically rigorous way in one space dimension. Moreover, we decide clearly the dependency of the constant of proportion upon the ratio of the length of two homopolymers and upon the quench depth. In the last section, we study the time evolution of the system. We first study the linear stability of spatially homogeneous steady state and derive the most unstable wavelength, if it is unstable. This is related to spinodal decomposition. Then, we numerically investigate the time evolution equation (the gradient flow of the free energy), and see that the free energy has many local minimizers and the system have some kind of sensitivity about initial data. (C) 1999 American Institute of Physics. [S1054-1500(99)02102-3]
\end{abstract}

In this paper we are mainly interested in characterizing the spatial periodic lamellar structure of a diblock copolymer. For the strong segregation limit, we completely determine the principal part of the asymptotic expansion of the period with respect to the interfacial thickness, which is a very small parameter, and give a mathematically rigorous proof. We also rigorously calculate the constant of proportionality and compute the dependence upon the ratio of two homopolymers, the quench depth and the long-range interaction. We consider this the first exact result for the problem. Second, we present some numerical experiments of the model, which is a certain discretization of the Cahn-Hilliard type partial differential equation with long-range interaction. We give a comparison between the rigorous result and the numerical simulation about the periodic structure of the strong segregation limit. Also, we show that a weak segregation limit on the numerical simulation has the finer period than the strong one, and the Gibbs energy functional has

\footnotetext{
${ }^{a)}$ Electronic mail: ohnishi@im.uec.ac.jp
}

many other local minimizers, all of which correspond to stable steady states. This demonstrates the richness of the spatial structures of the diblock copolymer melts.

\section{INTRODUCTION}

In this paper we consider the following functional:

$F_{\varepsilon, \sigma}(u)$

$=\int_{\Omega}\left(\frac{\varepsilon^{2}}{2}|\nabla u|^{2}+W(u)+\frac{\sigma}{2}\left|\left(-\Delta_{N}\right)^{-1 / 2}(u-\bar{u})\right|^{2}\right) d x$,

$\bar{u}=\frac{1}{|\Omega|} \int_{\Omega} u d x, \quad u \in H^{1}(\Omega)$,

under the following integral constraint:

$\bar{u}=m \quad(m$ is a given constant $)$,

where $\Omega$ is a smooth bounded domain in $\mathbf{R}^{n}, W(u)$ is a double-well potential with global minima $u= \pm 1$, typically of the form $\frac{1}{4}\left(u^{2}-1\right)^{2}, \varepsilon$ and $\sigma$ are positive constants, $H^{1}(\Omega)$ is the usual Sobolev space, and $\left(-\Delta_{N}\right)^{-1 / 2}$ is a frac- 
tional power of the Laplace operator under the zero flux boundary condition. [The underlying space for the Laplace operator is the subspace of $L^{2}(\Omega)$ orthogonal to constants. See Henry ${ }^{1}$ for details.] The third term is nonlocal, since $\left(-\Delta_{N}\right)^{-1 / 2}$ is, roughly speaking, an integral operator in $\Omega$. Without this term, (1.1) becomes a well-known functional from which we can derive the Allen-Cahn (nonconserved) and the Cahn-Hilliard (conserved) equations.

The functional (1.1) was first introduced in a different form by Refs. 2 and 3, then formulated like (1.1) in Ref. 4 in order to describe the microphase separation of diblock copolymers where two different homopolymers are connected, and this connectivity is responsible for introducing the longrange interaction, i.e., the nonlocal term of (1.1). The parameter $\sigma$ is inversely proportional to the square of the total chain length $N$ of the copolymer, and $\varepsilon$ represents the interfacial thickness at the bonding point assumed to be sufficiently small, and the average $m(-1<m<1)$ stands for the ratio of components of two homopolymers. In this paper we focus on a scaling regime $0<\varepsilon \ll 1$. The above micro constraint (connectivity) prevents a copolymer from forming a large domain, and hence the usual coarsening process stops at a certain stage of the mesoscopic level. Namely, (1.1) has the potential to have a variety of metastable states (local minimizers) with fine structures, which is not the case for the usual Cahn-Hilliard dynamics, although it has a long and interesting coarsening process. When one tries to minimize the functional (1.1), one easily see that there is a competition between the first gradient term and the third nonlocal term, assuming that $u$ is close to 1 or -1 off the interface. The first term wants to minimize the area of interface, however, the nonlocal term does not become small if $u$ takes 1 or -1 in a large domain. In order to make the third term small, $u$ has to oscillate rapidly around $\bar{u}$ (which increases the area of interface), in other words, if $u-\bar{u}$ converges to zero in a weak sense in $L^{2}(\Omega)$, it goes to zero because of the compactness of the operator $\left(-\Delta_{N}\right)^{-1 / 2}$. Thus, there should be an optimal domain size compromising these two opposite tendencies. The main problems in Ref. 4 were the following.

(I) Scaling law: Characterize the domain size in terms of $\varepsilon$ and $\sigma$.

(II) Morphology: Find a governing system of equations for the morphology of final states.

(III) Stability and selection mechanism of morphology.

The main aim of this paper is to answer rigorously the question (I) in one-dimensional space [i.e., $\Omega=(0,1)]$. Experimentally and numerically it is well-known in copolymer problems that the final asymptotic states prefer periodic structures such as lamellar, spherical, double-diamond geometries and so on (see, for instance, Refs. 5-7 and 3). Once one of the periodic structures is specified, it is not so difficult to determine the size of the periodic cell which minimizes the functional (1.1) within the specified class (see Ref. 2). Moreover, it is even possible, at least formally to derive a scaling law without specifying the periodic structure via dimensional analysis (see Ref. 3). Those arguments may be physically convincing, however, it is still unclear mathematically why such a scale is preferred, independent of the pre- cise structures. Our result in the next section determines completely the principal part of asymptotic expansion of the period and the free energy for the global minimizers in terms of $m, \varepsilon$ and $\sigma$.

In this paper we always assume the following conditions for the double-well potential $W$ in the functional (1.1).

(A1) The graph of $W(z)$ has the double tangent line with the tangent points $z= \pm 1+m^{\prime}$, where the above tangent line is defined as the graph of the function $r(u)=\alpha_{0} u+\beta_{0}$, for some $\alpha_{0} \in \mathbf{R}$ and $\beta_{0} \in \mathbf{R}$, and $m^{\prime}$ is the unique constant solving the equation: $W^{\prime}\left(m^{\prime}\right)=\alpha_{0}$ and is neither of the double tangent points:

(A2) $W \in C^{3}, W^{\prime \prime}( \pm 1)>0, W^{\prime \prime}(0)<0$.

(A3) $W^{\prime}(z)<\alpha_{0}$, if $-\infty<z<-1+m^{\prime}$ or $m^{\prime}<z<1+m^{\prime}$, and there exists a unique $z_{+}$such that $W^{\prime \prime}\left(z_{+}\right)=0$.

(A4) $W^{\prime}(z)>\alpha_{0}$, if $-1+m^{\prime}<z<m^{\prime}$ or $1+m^{\prime}<z<\infty$, and there exists a unique $z_{-}$such that $W^{\prime \prime}\left(z_{-}\right)=0$.

(A5) $\lim _{z \rightarrow \pm \infty}\left|W^{\prime}(z)\right|>\alpha_{0}$.

The interval $\left(z_{-}, z_{+}\right)$is called a "spinodal region," and $\left(-1, z_{1}\right) \cup\left(z_{+}, 1\right)$ is called a "metastable region (or miscibility gap)". We briefly explain the reason why we separate the interval $(-1,1)$. If the average density $m$ is in the spinodal region, the homogeneous stationary state $(u \equiv m)$ is exponentially unstable, but if the average density $m$ is in the metastable region, it is exponentially stable. (This statement will be proven rigorously in the last section in this paper.) The evolution of the system is therefore different between the former case and the latter.

We now easily see $W^{\prime}\left(m^{\prime}\right)=\alpha_{0}$ from (A2), (A3) and (A4). We define $\widetilde{W}(\widetilde{z})$ by

$$
\widetilde{W}(\widetilde{z})=W\left(\widetilde{z}+m^{\prime}\right)-\left(\alpha_{0}\left(\widetilde{z}+m^{\prime}\right)+\beta_{0}\right), \quad \widetilde{z}=z-m^{\prime} .
$$

We set the new functional $\widetilde{F}_{\varepsilon, \sigma}(\widetilde{u})$,

$\widetilde{F}_{\varepsilon, \sigma}(\widetilde{u})$

$$
=\int_{\Omega}\left(\frac{\varepsilon^{2}}{2}|\nabla \widetilde{u}|^{2}+\widetilde{W}(\widetilde{u})+\frac{\sigma}{2}\left|\left(-\Delta_{N}\right)^{-1 / 2}(\widetilde{u}-\tilde{\tilde{u}})\right|^{2}\right) d x,
$$

under the corresponding integral constraint:

$$
\frac{1}{|\Omega|} \int_{\Omega} \widetilde{u} d x=m-m^{\prime} .
$$

Due to the constraint, we compute

$$
\begin{aligned}
F_{\varepsilon, \sigma}(u)= & \widetilde{F}_{\varepsilon, \sigma}(\widetilde{u})+\left(\alpha_{0} m+\beta_{0}\right)|\Omega|, \\
& \text { if } u \text { is in the admissible space. }
\end{aligned}
$$

We therefore see the set of all the critical points of $F_{\varepsilon, \sigma}$ in the admissible space of $u$ mapped one to one and onto the set of all critical points of $\widetilde{F}_{\varepsilon, \sigma}$ in the corresponding admissible space of $\widetilde{u}$. Moreover, we realize that $\widetilde{W}$ satisfies the following. 
(A1') $\tilde{W}(\widetilde{z})=0$, if $\widetilde{z}= \pm 1$, and $\widetilde{W}(\widetilde{z})>0$, otherwise.

$\left(\mathrm{A} 2^{\prime}\right) \widetilde{W} \in C^{3}, \widetilde{W}^{\prime \prime}( \pm 1)>0, \widetilde{W}^{\prime \prime}(0)<0$.

(A3') $\widetilde{W}^{\prime}(\widetilde{z})<0$, if $-\infty<\widetilde{z}<-1$ or $0<\widetilde{z}<1$, and there exists a unique $\widetilde{z}_{+}$such that $\widetilde{W}^{\prime \prime}\left(\widetilde{z}_{+}\right)=0$.

(A4') $\widetilde{W}^{\prime}(\widetilde{z})>0$, if $-1<\widetilde{z}<0$ or $1<\widetilde{z}<\infty$, and there exists a unique $\widetilde{z}_{-}$such that $\widetilde{W}\left(\widetilde{z}_{-}\right)=0$.

(A5') $\lim _{\tilde{z} \rightarrow \pm \infty}\left|\tilde{W}^{\prime}(\widetilde{z})\right|>0$.

Note that we assume neither the symmetry of $W$ nor $\widetilde{W}$.

We now let our concentration be upon the problem in one space dimension. Set $\Omega:=(0,1)$, and define the admissible function space $\operatorname{Ad}_{m}$ by

$$
\operatorname{Ad}_{m}:=\left\{u \in H^{1}(0,1) ; \int_{0}^{1} u(t) d t=m\right\} .
$$

We state our main result.

Theorem 1.1: Assume that (A1), (A2), (A3), (A4) and (A5) are satisfied. For given $z_{-}<m<m^{\prime}<z_{+}$and $\sigma>0$, there exists a constant $\varepsilon_{0}>0$ such that, if $0<\varepsilon<\varepsilon_{0}$ and if $u^{\varepsilon, \sigma, m}$ is a global minimizer of $F_{\varepsilon, \sigma}$ in $\operatorname{Ad}_{m}$ and a normal $n$-layered solution, then $u^{\varepsilon, \sigma, m}$ is the generically unique global minimizer and the period $P^{\varepsilon, \sigma, m}$ of $u^{\varepsilon, \sigma, m}$ satisfies

$$
P^{\varepsilon, \sigma, m}=2\left(\frac{6 \sqrt{2} A}{\left(1-\left(m-m^{\prime}\right)^{2}\right)^{2}} \frac{\varepsilon}{\sigma}\right)^{1 / 3}+O\left(\varepsilon^{2 / 3}\right),
$$

and

$$
\begin{aligned}
F_{\varepsilon, \sigma}\left(u^{\varepsilon, \sigma, m}\right)= & \alpha_{0} m+\beta_{0}+\frac{\sigma}{8} \\
& \times\left(6 \sqrt{2}\left(1-\left(m-m^{\prime}\right)^{2}\right) A \frac{\varepsilon}{\sigma}\right)^{2 / 3}+O\left(\varepsilon^{4 / 3}\right),
\end{aligned}
$$

where

$$
A=2 \int_{-1}^{1} \sqrt{\widetilde{W}(y)} d y .
$$

Remarks:

(1) For the definition of a normal $n$-layered solution, we refer to the head part of Sec. III of this paper.

(2) In Theorem 1.1, we completely determine the principal term of the asymptotic expansion of the spatial period with respect to $\varepsilon$ (interfacial thickness) by mathematically rigorous way, namely in this paper we estimate rigorously the higher order error term with respect to $\varepsilon$, and moreover determine the constant of proportion and derive its $m$ and $A$ dependency clearly. Both our model and the one in Ref. 2 are based on the same thermodynamic principle (Gibbs thermodynamic equilibrium theory). We remark that they lead to $P^{\varepsilon, \sigma, m} \propto(\varepsilon / \sigma)^{1 / 3}$ near $m=0$ by the way of theoretical physics in Ref. 2 .

(3) In Fig. 2 in Ref. 8, the domain size of the lamellar structure in a chemical experiment (Styrene-2-vinylpyridine diblock copolymer) is compared with several theoretical values, one of which is contained in Ref. 2, as $m$ is nearly equal to 0 . The theoretical value in Ref. 2 does not agree with the experimental value very much. Our theoretical value may not also agree with it, although we have no chemical experiment in which investigating $m$ and $A$ dependency of the spatial period.

(4) The assumption that $u^{\varepsilon, \sigma, m}$ is a normal $n$-layered solution seems to be technical because all the global minimizers are so in the numerical calculations in Sec. IV. In fact, if $W$ has the symmetry and $m-m^{\prime}=0$, then it is proved in Ref. 9. By Lemma 2.1, in the symmetric case, our problem is mathematically equivalent to the one considered in Ref. 9, although the background of physics of our one is different from the one in Ref. 9.

We should explain in detail the mathematical connection between our problem and the one of Müller in Ref. 9. In his context, the functional is given by

$$
I^{\epsilon}(v)=\int_{0}^{1}\left(\epsilon^{2}\left|v_{x x}\right|^{2}+W\left(v_{x}\right)+v^{2}\right) d x,
$$

with the periodic boundary conditions or the homogeneous Dirichlet conditions. Both these boundary conditions are corresponding to the integral constraint with $m=0$ in our context (see Lemma 2.1 proved later in Sec. II). Moreover, by the same change of variables used to get (1.3), we have

$$
\widetilde{I}^{\epsilon}(\widetilde{v})=\int_{0}^{1}\left(\epsilon^{2} \widetilde{v}_{x x}^{2}+\widetilde{W}\left(\widetilde{v}_{x}\right)+\left(\widetilde{v}+m^{\prime} x\right)^{2}\right) d x,
$$

with the suitable boundary conditions. In view of Lemma 2.1 , on the other hand, make the following change of variables in the functional (1.3) with the constraint of $m=0$ :

$$
\widetilde{v}(x)=\int_{0}^{x} \widetilde{u}(y) d y, \quad \varepsilon=\sqrt{2} \epsilon, \quad \sigma=2,
$$

and we get the same functional as (1.7). Theorem 1.1 is therefore regarded as an extension Müller's result in Ref. 9 to the nonsymmetric double-well potential case. This extension has difficulty derived from considering the integral constraint explicitly in making a periodic candidate, because the global minimizer does not have the symmetry with respect to the $x$-axis in case of $m \neq 0$, unlike Müller's one. But, due to it, we assume the spatial periodicity or antiperiodicity of a global minimizer. We manage to overcome the difficulty by the utilizing the "simple solution" of Carr, Gurtin and Slemrod, ${ }^{10}$ in which they investigate the steady states of the Cahn-Hilliard equation, to construct the periodic candidate, although their case does not have the third nonlocal interaction, of course. Our main effort is spent in estimating a global minimizer of the adequately rescaled functional, which is the candidate, by using properties of the "simple solution" and in analogous way in Ref. 9.

\section{PRELIMINARY}

At first we set $\Omega=(0,1)$ and

$$
\widetilde{\varepsilon}=\frac{\varepsilon}{\sqrt{2}}, \quad \widetilde{\sigma}=\frac{\sigma}{2}, \quad \widetilde{m}=m-m^{\prime},
$$

and put them into (1.3). We therefore get 
$\widetilde{F}_{\widetilde{\varepsilon}, \widetilde{\sigma}}(\widetilde{u})$

$$
=\int_{0}^{1}\left(\widetilde{\varepsilon}^{2}\left|\widetilde{u}_{x}\right|^{2}+\widetilde{W}(\widetilde{u})+\widetilde{\sigma}\left|\left(-\Delta_{N}\right)^{-1 / 2}(\widetilde{u}-\tilde{\tilde{u}})\right|^{2}\right) d x,
$$

and the corresponding integral constraint:

$$
\int_{0}^{1} \tilde{u} d x=\widetilde{m} .
$$

In order to simplify the notation, in what follow $\mathrm{S}$ we write $F, \varepsilon, W, \sigma, m$ and $u$ instead of $\widetilde{F}, \widetilde{\varepsilon}, \widetilde{W}, \widetilde{\sigma}, \widetilde{m}$ and $\widetilde{u}$, respectively, as we think we are not thrown into confusion. From here to the end of the proof of Theorem 1.1, we consider the functional (2.1) without an overtilde under the constraint, with $W$ satisfying $\left(\mathrm{A} 1^{\prime}\right),\left(\mathrm{A} 2^{\prime}\right),\left(\mathrm{A} 3^{\prime}\right),\left(\mathrm{A} 4^{\prime}\right)$ and $\left(\mathrm{A} 5^{\prime}\right)$.

We utilize the following notations for an $L^{2}$ inner product and norm:

$$
(u, v)_{L^{2}}=\int_{a}^{b} u(t) v(t) d t, \quad\|u\|_{L^{2}}=\sqrt{\int_{a}^{b} u(t)^{2} d t},
$$

for any $u, v \in L^{2}(a, b)$ and $(a, b) \subset \mathbf{R}$ under consideration. We prove a lemma which means that the third term of the functional (2.1) is represented by an indefinite integral.

Lemma 2.1: For any $u \in L^{2}(a, b)$ with $\int_{a}^{b} u d x=m$ and $m$ is a given constant,

$$
\int_{a}^{b}\left|\left(-\Delta_{N}\right)^{-1 / 2}(u-m)\right|^{2} d x=\int_{a}^{b}\left|\int_{a}^{x}(u(t)-m) d t\right|^{2} d x .
$$

Proof: We denote $u^{\prime}(x)$ as $d u / d x$. If $v=\left(-\Delta_{N}\right)^{-1}(u$ $-m), v$ satisfies

$$
-v^{\prime \prime}=u-m, \text { in }(a, b), v^{\prime}(a)=v^{\prime}(b)=0 .
$$

Hence

$$
\int_{a}^{x}(u(t)-m) d t=-\int_{a}^{x} v^{\prime \prime}(t) d t=-v^{\prime}(x) .
$$

On the other hand, we calculate

$$
\begin{aligned}
\left\|\left(-\Delta_{N}\right)^{-1 / 2}(u-m)\right\|_{L^{2}}^{2} & =\left(\left(-\Delta_{N}\right)^{-1}(u-m), u-m\right)_{L^{2}} \\
& =\left(v,-v^{\prime \prime}\right)_{L^{2}}=\left\|v^{\prime}\right\|_{L^{2}}^{2} .
\end{aligned}
$$

As combining two, we get the result.

The idea of proving Theorem 1.1 is to construct a periodic candidate for the minimizer and then to show that it is optimal and that any other minimizer has to be periodic as well. This idea is the same as in Ref. 9. We consider the rescaling functional in view of Lemma 2.1 with $(a, b)$ $=\left(-\frac{1}{2}, \frac{1}{2}\right)$ :

$$
\begin{aligned}
I_{l}(u)= & \int_{-1 / 2}^{1 / 2}\left(\frac{\varepsilon^{2}}{l} u_{x}^{2}+l W(u)+\sigma l^{3}\right. \\
& \left.\times\left|\int_{-1 / 2}^{x}(u(t)-m) d t\right|^{2}\right) d x,
\end{aligned}
$$

where we have used $u_{x}$ as $d u / d x$. If $m=0$ and there is the symmetry, we are considering the same problem as in Ref. 9 by the change of variables:

$$
v(x)=\int_{-1 / 2}^{x} u(t) d t .
$$

We therefore see Theorem 1.1 in Sec. I directly from the main result in Ref. 9. But we wish to consider it in the general conditions without the symmetry.

To make the half of a candidate, consider the following quantities:

$$
\begin{aligned}
& E_{m}(l)=\min \left\{I_{l}(u) ; u \in H_{\text {adm }}\right\}, \\
& \widetilde{E}_{m}(l)=\min \left\{I_{l}(u) ; u \in H_{\text {sym }}\right\},
\end{aligned}
$$

where the admissible function spaces are defined, respectively, by

$$
H_{\text {adm }}=\left\{u \in H^{1}\left(-\frac{1}{2}, \frac{1}{2}\right) ; \int_{-1 / 2}^{-1 / 2} u d x=m,\right.
$$

and there are $p_{m}^{ \pm} \in\left(-\frac{1}{2}, \frac{1}{2}\right)$ such that $u\left(p_{m}^{ \pm}\right)=0$,

$$
\begin{aligned}
& u(x) \leqslant m \text { in }\left(-\frac{1}{2}, p_{m}^{-}\right) \cup\left(p_{m}^{+}, \frac{1}{2}\right), \\
& \left.u(x) \geqslant m \text { in }\left(p_{m}^{-}, p_{m}^{+}\right)\right\}, \\
& H_{s y m}=\left\{u \in H_{a d m} ; u(-x)=u(x)\right\} .
\end{aligned}
$$

Convention: We promise that $C$ denotes any constant independent of $m, \sigma, l$ and $\varepsilon$ in this paper under the rule that, if necessary, we exchange it for a more adequate one.

We now consider the Euler-Lagrange equation of this rescaling variational problem.

Lemma 2.2: Let $u \in H^{1}\left(-\frac{1}{2}, \frac{1}{2}\right)$ be a critical point of $I_{l}(u)$ subject to the constraint

$$
\int_{-1 / 2}^{-1 / 2} u d x=m
$$

Then $u$ belongs at least to $C^{4}\left(\left[-\frac{1}{2}, \frac{1}{2}\right]\right)$ and satisfies the following equation:

$$
\begin{aligned}
& -\frac{2 \varepsilon^{2}}{l} u_{x x}+l W^{\prime}(u)+2 \sigma l^{3} v=\mu \quad \text { in }\left(-\frac{1}{2}, \frac{1}{2}\right), \\
& -v_{x x}=u-m, \quad \text { in } \quad\left(-\frac{1}{2}, \frac{1}{2}\right), \\
& u_{x}\left( \pm \frac{1}{2}\right)=v_{x}\left( \pm \frac{1}{2}\right)=0 \\
& \int_{-1 / 2}^{1 / 2} u(t) d t=m, \quad \int_{-1 / 2}^{1 / 2} v(t) d t=0,
\end{aligned}
$$

where $\mu$ is the Lagrange multiplier, which is a constant dependent upon a critical point.

Proof: The proof is a standard argument of the variational problems with constraint. As we calculate the Gâteaux derivative at $u$ to the direction $\phi$ with $\int_{-1 / 2}^{1 / 2} \phi d x=0$, we have 


$$
\begin{aligned}
0=I_{l}^{\prime}(u) \phi= & \int_{-1 / 2}^{1 / 2}\left(\frac{2 \varepsilon^{2}}{l} u_{x} \phi_{x}+l W^{\prime}(u) \phi+2 \sigma l^{3}\right. \\
& \left.\times(-\Delta N)^{-1 / 2}(u-m) \cdot\left(-\Delta_{N}\right)^{-1 / 2}(\phi)\right) d x .
\end{aligned}
$$

By the condition $\left(\mathrm{A} 2^{\prime}\right)$, the standard regularity theory and the Sobolev imbedding theorem, $u$ belongs at least to $C^{4}\left(\left[-\frac{1}{2}, \frac{1}{2}\right]\right)$. By integrating by parts,

$$
\begin{aligned}
I_{l}^{\prime}(u) \phi= & \int_{-1 / 2}^{1 / 2}\left\{\left(-\frac{2 \varepsilon^{2}}{l} u_{x x}+l W^{\prime}(u)+2 \sigma l^{3}\right.\right. \\
& \left.\left.\times\left(-\Delta_{N}\right)^{-1}(u-m)\right) \phi\right\} d x+\left[\frac{2 \varepsilon^{2}}{l} u_{x} \phi\right]_{-1 / 2}^{1 / 2} .
\end{aligned}
$$

Here, by the constraint, $\phi$ is any function in the function space $V$ defined by

$$
V=\left\{\phi \in H^{1}\left(-\frac{1}{2}, \frac{1}{2}\right) ; \quad \int_{-1 / 2}^{1 / 2} \phi d x=0\right\},
$$

and we use the self-adjointness of $\left(-\Delta_{N}\right)^{-1 / 2}$ on $V$.

Now take $\phi$ in the subspace $V_{0}$ defined by

$$
V_{0}=\left\{\phi \in V ; \phi\left( \pm \frac{1}{2}\right)=0\right\}
$$

and we get the equation satisfied by $u$, because $V_{0}$ is densely imbedded in $V$ in $L^{2}$-topology. By arbitrariness of the values of $\phi\left( \pm \frac{1}{2}\right)$ for $\phi \in V$, we next obtain the natural boundary condition $u\left( \pm \frac{1}{2}\right)=0$. Finally, set $v=\left(-\Delta_{N}\right)^{-1}(u-m)$ and we get the equation, the boundary condition and the integral constraint of $v$.

The classical theory of the calculus of variations asserts that $u$ attaining $E_{m}(l)$ is a critical point of $I_{l}$ in $H^{1}\left(-\frac{1}{2}, \frac{1}{2}\right)$ with $\int_{-1 / 2}^{1 / 2} u(x) d x=m$ and that there at least exists such a global minimizer. We denote $\underline{u}$ as any one of such global minimizers. By differentiating the Euler-Lagrange equation twice in $x$, we obtain

$$
\frac{2 \varepsilon^{2}}{l} \underline{u}_{x x x x}-l\left(W^{\prime}(\underline{u})\right)_{x x}+2 \sigma l^{3} 2(\underline{u}(x)-m)=0 .
$$

If there is a point $x_{0}$ at which $u_{x}=u_{x x}=u_{x x x}=u_{x x x x}=0$, then $\underline{u}(x)=$ the constant $\left(=\underline{u}\left(x_{0}\right)\right)$.

Now let us prepare for investigating some properties of $\underline{u}$. As in Ref. 10, we define the associated Gibbs function $\Phi_{\lambda}$ in the rescaled problem by

$$
\Phi_{\lambda}(y)=W(y)-\lambda y,
$$

for any $y \in \mathbf{R}$ and a constant $\lambda$, and consider the solution $q$ of the ordinary differential equation;

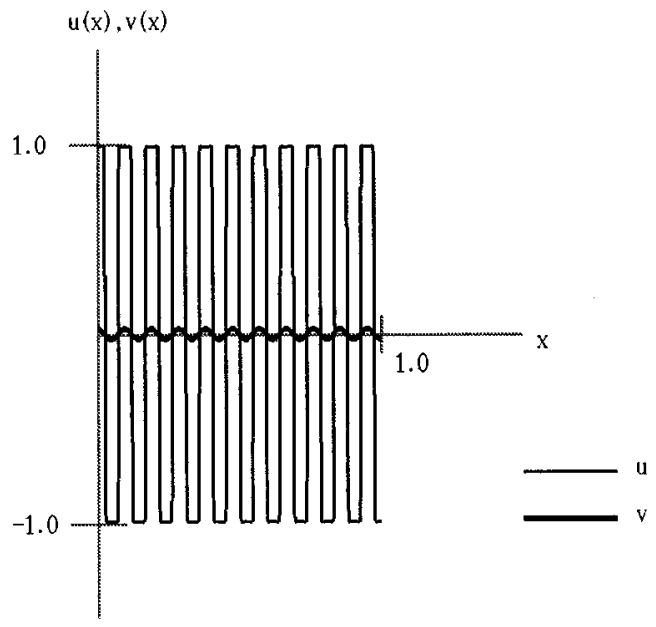

FIG. 1. Shape of the global minimizer as $m=0.0$.

$$
\begin{aligned}
& 2 q^{\prime \prime}(z)=\Phi_{\lambda}^{\prime}(q(z)) \text { in }\left(-\frac{1}{2}, 0\right), \\
& q^{\prime}\left(-\frac{l}{2 \varepsilon}\right)=q^{\prime}(0)=0, \\
& \int_{-l / 2 \varepsilon}^{0} q(z) d z=\frac{l m}{2 \varepsilon} .
\end{aligned}
$$

The first equation of (2.4) has the first integral

$$
q^{\prime}(z)^{2}=\Phi_{\lambda}(q(z))-\nu,
$$

with $\nu$ a constant. By the boundary condition of (2.4), we see

$$
\Phi_{\lambda}\left(q_{1}\right)=\Phi_{\lambda}\left(q_{2}\right)=\nu
$$

where we have set $q_{1}=q(-l / 2 \varepsilon)$ and $q_{2}=q(0)$.

Now the next Lemma is the same as Proposition 2.1 in Ref. 10. Note that $\lambda=0$ is the Maxwell line. (cf. Fig. 1).

Lemma 2.3 (properties of the Gibbs function): The mapping $(q, \lambda) \mapsto \Phi_{\lambda}(q)$ is in the $C^{3}$-class. Further, for each $\lambda$ $\in(\underline{\lambda}, \bar{\lambda})$,

- $\Phi_{\lambda}$ has exactly three critical points $\alpha_{\lambda}<\zeta_{\lambda}$ $<\beta_{\lambda}: \Phi_{\lambda}^{\prime}\left(\alpha_{\lambda}\right)=\Phi_{\lambda}^{\prime}\left(\zeta_{\lambda}\right)=\Phi_{\lambda}^{\prime}\left(\beta_{\lambda}\right)$

- $\Phi$ is strictly decreasing on $\left(-\infty, \alpha_{\lambda}\right) \cup\left(\zeta_{\lambda}, \beta_{\lambda}\right)$, strictly increasing on $\left(\alpha_{\lambda}, \zeta_{\lambda}\right) \cup\left(\beta_{\lambda}, \infty\right)$;

- $\Phi_{\lambda}\left(\alpha_{\lambda}\right)<\Phi_{\lambda}\left(\beta_{\lambda}\right)$ for $\lambda<0, \Phi_{\lambda}\left(\alpha_{\lambda}\right)>\Phi_{\lambda}\left(\beta_{\lambda}\right)$ for $\lambda$ $>0$, and $\Phi_{0}(-1)=\Phi_{0}(1)$.

Denote $q(x)$ by the antisymmetric extension in $\left(-\frac{1}{2}, \frac{1}{2}\right)$ of the solution of (2.4), and also define $q_{l}$ by

$$
q_{l}(x)=q\left(\frac{l x}{\varepsilon}\right)
$$

Moreover, define $k_{m} \in\left(0, \frac{1}{2}\right)$ as the real number $k_{m}$ $\left(=k_{m}(\varepsilon, \sigma, l)\right)$ such that $q_{l}\left(k_{m}\right)=m$. We remark that $q_{l}$ is the antisymmetric extension of the "simple solution", in Ref. 10 , in which they investigate it in detail. The "simple solution"' is the global minimizer of the functional $F_{\varepsilon, 0}$, which is without a nonlocal interaction, and this satisfies the usual stationary Cahn-Hilliard equation: 


$$
\begin{aligned}
& \frac{2 \varepsilon^{2}}{l^{2}}\left(q_{l}\right)_{x x}=\Phi_{\lambda}^{\prime}\left(q_{l}\right), \text { in }\left(-\frac{1}{2}, 0\right), \\
& \left(q_{l}\right)_{x}\left(-\frac{1}{2}\right)=\left(q_{l}\right)_{x}(0)=0, \\
& \int_{-1 / 2}^{0} q_{l}(x) d x=\frac{m}{2} .
\end{aligned}
$$

The main theorem (Theorem 3.1) in Ref. 10 means the next proposition in the context of (2.6).

Proposition 2.4 (the Maxwell solution of the usual Cahn-Hilliard equation): Given $l>0, \delta>0$, there are an $\varepsilon_{\delta}$ and a neighborhood $N_{\delta}$ of the Maxwell point $(0,0)$, such that for $\varepsilon \in\left(0, \varepsilon_{\delta}\right)$ and $m \in[-1+\delta, 1-\delta]$, there uniquely exists a simple solution with a corresponding admissible pair $(\lambda, \nu) \in N_{\delta}$. Further, there are constants $C=C_{\delta}>0$ and $D$ $=D_{\delta}>0$ such that

$$
|(\lambda, \nu)|,\left|q_{1}-(-1)\right|,\left|q_{2}-1\right|,
$$

are in $O\left(e^{-C l / \varepsilon}\right)$ and

$$
\left|k_{m}-\left(-\frac{1+m}{4}\right)\right|=D \frac{\varepsilon}{l}+o\left(\frac{\varepsilon}{l}\right),
$$

as $\varepsilon \rightarrow 0$, uniformly for $m \in[-1+\delta, 1-\delta]$.

The fourth convergence is not stated explicitly in Ref. 10 , but it is easy to see it because the width of the internal layer is in $O(\varepsilon / l)$.

To prove the next lemma, we now define some constants:

$$
\begin{aligned}
& A\left(q_{1}, q_{2}\right)=2 \int_{q_{1}}^{q_{2}} \sqrt{W(t)} d t \\
& K\left(q_{1}, q_{2}, k_{m}\right) \\
& =2\left(\frac{1}{3}\left(q_{1}-m\right)^{2}\left(\frac{1}{2}-k_{m}\right)^{3}+\left(q_{1}-m\right)^{2}\left(\frac{1}{2}-k_{m}\right)^{2} k_{m}\right. \\
& \left.\quad-\left(m-q_{1}\right)\left(q_{2}-m\right)\left(\frac{1}{2}-k_{m}\right) k_{m}^{2}+\frac{1}{3}\left(q_{2}-m\right)^{2} k_{m}^{3}\right) .
\end{aligned}
$$

We remark, noting that $A$ is defined in Theorem 1.1, that

$$
\begin{aligned}
& A(-1,1)=A, \\
& K\left(q_{1}, q_{2}, k_{m}\right)=\frac{1}{48}\left(1-m^{2}\right)^{2}+C^{(m)} \frac{\varepsilon}{l}+o\left(\frac{\varepsilon}{l}\right),
\end{aligned}
$$

as $\varepsilon$ tends to 0 , uniformly for $m \in[-1+\delta, 1-\delta]$, where $C^{(m)}$ is a constant dependent upon $m$ and $D$.

We now give the best possible upper bound of $\underline{u}$. It is simple, but is important.

Lemma 2.5: For a given $\sigma>0,-1<m<1$, and $0<l$ $\leqslant 1$, there exists $\varepsilon_{0}>0$ such that, if $\varepsilon \in\left(0, \varepsilon_{0}\right)$, then

$$
\begin{aligned}
E_{m}(l) \leqslant \widetilde{E}_{m}(l) \leqslant & 2 A \varepsilon+\frac{1}{48}\left(1-m^{2}\right)^{2} \sigma l^{3}+C^{(m)} \sigma l^{2} \varepsilon \\
& +o(\varepsilon) \sigma l^{2} .
\end{aligned}
$$

Proof: For any $(a, b) \subset\left(-\frac{1}{2}, \frac{1}{2}\right)$,

$$
\begin{aligned}
\int_{a}^{b}\left(\frac{\varepsilon^{2}}{l} u_{x}^{2}+l W(u)\right) d x & \geqslant 2 \int_{a}^{b} \sqrt{\varepsilon^{2} u_{x}^{2} W(u)} d x \\
& \geqslant 2 \varepsilon \int_{a}^{b} u_{x} \sqrt{W(u)} d x .
\end{aligned}
$$

When the equality holds, $\left(\varepsilon^{2} / l\right) u_{x}^{2}=l W(u)$. We note that $q_{l}$ is in the function space $H_{a d m}$ and that it is the function at which the equality in the inequality (2.8) holds approximately in the error of order $e^{-C l / \varepsilon}$, because of its way of construction and Proposition 2.4. For the third term of $I_{l}$, we compute

$$
\begin{aligned}
\int_{-1 / 2}^{1 / 2}\left|\int_{-1 / 2}^{x}\left(q_{l}(t)-m\right) d t\right|^{2} d x \\
\leqslant 2\left(\int_{-1 / 2}^{-k_{m}}\left(q_{1}-m\right)^{2}\left(x+\frac{1}{2}\right)^{2} d x\right. \\
\quad+\int_{-k_{m}}^{0}\left(\left(q_{1}-m\right)\left(-k_{m}+\frac{1}{2}\right)\right. \\
\left.\left.\quad+\left(q_{2}-m\right)\left(x+k_{m}\right)\right)^{2} d x\right) \\
=K\left(q_{1}, q_{2}, k_{m}\right) .
\end{aligned}
$$

Therefore,

$$
\begin{aligned}
E(l) \leqslant & I_{l}\left(q_{l}\right) \\
= & 2 \int_{-1 / 2}^{1 / 2} \sqrt{\varepsilon^{2}\left(q_{l}\right)_{x}^{2} W\left(q_{l}\right)} d x \\
& +\sigma l^{3} \int_{-1 / 2}^{1 / 2}\left|\int_{-1 / 2}^{x}\left(q_{l}(t)-m\right) d t\right|^{2} d x+O\left(e^{-C_{l} / \varepsilon}\right) \\
\leqslant & 2 \varepsilon \int_{-1 / 2}^{1 / 2} \sqrt{W\left(q_{l}\right)}\left|\left(q_{l}\right)_{x}\right| d x+K\left(q_{1}, q_{2}, k_{m}\right) \sigma l^{3} \\
& +O\left(e^{-C_{l} / \varepsilon}\right) \leqslant 2 A(-1,1) \varepsilon+K\left(-1,1, \frac{1+m}{4}\right) \sigma l^{3} \\
& +C^{(m)} \sigma l^{2} \varepsilon+o(\varepsilon) \sigma l^{2} .
\end{aligned}
$$

We thus get (2.7).

Furthermore, we define the following constants:

$$
\begin{aligned}
& M_{m}^{(1)}= \operatorname{meas}\left\{x \in\left(-\frac{1}{2}, \frac{1}{2}\right) ; \underline{u}(x) \leqslant \frac{-1+m}{2}\right\}, \\
& M_{m}^{(2)}=\operatorname{meas}\left\{x \in\left(-\frac{1}{2}, \frac{1}{2}\right) ; \underline{u}(x) \geqslant \frac{1+m}{2}\right\}, \\
& M_{m}=M_{m}^{(1)}+M_{m}^{(2)}, \quad B(\tau, m)=2 \int_{(\tau+m) / 2}^{m} \sqrt{W(t)} d t, \\
& C\left(-1,1, M_{m}, m, \sigma\right) \\
&=\left(1-M_{m}\right) \min \left(W\left(\frac{-1+m}{2}\right), W\left(\frac{1+m}{2}\right)\right) \\
& \quad+\sigma L\left(-1,1, M_{m}^{(1)}, M_{m}^{(2)}, m\right),
\end{aligned}
$$




$$
\begin{aligned}
L\left(a, b, M_{m}^{(1)}, M_{m}^{(2)}, m\right) & \begin{aligned}
3 & \left.\frac{a-m}{2}\right)^{2}\left(\left(\frac{1}{2}\right)^{3}-\left(\frac{1}{2}-M_{m}^{(1)}\right)^{3}\right)+\left(\frac{a-m}{4}\right)^{2} M_{m}^{(2)} \\
& -\frac{(m-a)(b-m)}{2}\left(M_{m}^{(2)}\right)^{2}+\frac{1}{12}(b-m)^{2}\left(M_{m}^{(2)}\right)^{3} .
\end{aligned}
\end{aligned}
$$

We remark that $\min _{\tau \in(0,1)} C(-1,1, \tau, m, \sigma)$ does not vanish.

The next lemma means a lower bound of the energy of $\underline{u}$.

Lemma 2.6: For a given $\sigma>0,-1<m<1,0<l \leqslant 1$, and $0<\varepsilon \leqslant 1$, the following estimates hold:

$$
\begin{aligned}
E_{m}(l) \geqslant & \min \left(B(-1, m) \varepsilon,|B(1, m)| \varepsilon, l W\left(\frac{-1+m}{2}\right),\right. \\
& \left.l W\left(\frac{1+m}{2}\right)\right), \\
E_{m}(l) \geqslant & C\left(-1,1, M_{m}, m, \sigma\right) \sigma l^{3} .
\end{aligned}
$$

Proof: Let $\underline{x}$ and $\bar{x}$ be a minimum point and a maximum point of $\underline{u}$, respectively. If $(-1+m) / 2 \leqslant \underline{u}(\underline{x})$ and $(1$ $+m) / 2 \geqslant \underline{u}(\bar{x})$, then

$$
\begin{aligned}
I_{l}(\underline{u}) & \geqslant \int_{-1 / 2}^{1 / 2} l W(\underline{u}) d x \\
& \geqslant l \min \left(W\left(-\frac{1+m}{2}\right), W\left(\frac{1+m}{2}\right)\right) .
\end{aligned}
$$

Otherwise, for example, if we may assume that $(-1+m) / 2$ $>\underline{u}(\underline{x})$, then

$$
\begin{aligned}
& \int_{-1 / 2}^{1 / 2}\left(\frac{\varepsilon^{2}}{l} \underline{u}_{x}^{2}+l W(\underline{u})\right) d x \\
& \geqslant 2 \varepsilon\left|\int_{\underline{x}}^{p_{m}} \sqrt{W(\underline{u})} \underline{u}_{x} d x\right| \geqslant \varepsilon B(-1, m) .
\end{aligned}
$$

In the other cases, the similar argument works, and, after all, we get (2.9).

Finally we prove (2.10). We remark that, as $l \leqslant 1$,

$$
\begin{aligned}
l \int_{-1 / 2}^{1 / 2} W(\underline{u}) d x \geqslant & l^{3}\left(1-M_{m}\right) \\
& \times \min \left(W\left(\frac{-1+m}{2}\right), W\left(\frac{1+m}{2}\right)\right) .
\end{aligned}
$$

By the definition of $M_{m}^{(1)}$ and $M_{m}^{(2)}$, we compute

$$
\begin{aligned}
& \int_{-1 / 2}^{1 / 2}\left|\int_{-1 / 2}^{x}(u(t)-m) d t\right|^{2} d x \\
& \geqslant \int_{-M_{m}^{(1)}}^{0}\left(\frac{-1-m}{2}\right)^{2}\left(x+\frac{1}{2}\right)^{2} d x \\
& \quad+\int_{0}^{M_{m}^{(2)}}\left(\frac{-1-m}{4}+\left(\frac{1-m}{2}\right) x\right)^{2} d x \\
& =L\left(-1,1, M_{m}^{(1)}, M_{m}^{(2)}, m\right) .
\end{aligned}
$$

Noting that $l \leqslant 1$, we obtain

$$
E_{m}(l) \geqslant C\left(-1,1, M_{m}, m, \sigma\right) \sigma l^{3} .
$$

We complete the proof.

We remark that $\underline{u}$ is a two-layered pattern of the singular perturbed system treated in Ref. 11 (also refer to Fife, ${ }^{12}$ Mimura, Tabata and Hosono, ${ }^{13}$ Ito $^{14}$ and Nishiura and Fujii ${ }^{15}$ ), because of the conditions of $W$ and $m$. We therefore see the width of the internal layer be in $O(\epsilon / l)$ and the internal layers be near $\pm(1+m) / 4$.

More precisely we possess the following.

Proposition 2.7: Assume that $W$ satisfies $\left(A 1^{\prime}\right),\left(A 2^{\prime}\right)$, $\left(A 3^{\prime}\right),\left(A 4^{\prime}\right)$, and $\left(A 5^{\prime}\right)$, and that $m \in\left(z_{-}, z_{+}\right)$. There exist constants $C$ and $c_{0}$ such that, if

$$
l \leqslant c_{0} \text { and } \varepsilon \leqslant c_{0} \frac{l}{|\log l|},
$$

then

$$
\begin{aligned}
& || \underline{u}(x)|-1| \leqslant C \sigma l^{2}, \quad \frac{\varepsilon}{l}\left|\underline{u}_{x}(x)\right| \leqslant C \sigma l^{2}, \\
& \text { and } \frac{\varepsilon^{2}}{l^{2}}\left|\underline{u}_{x x}(x)\right| \leqslant C \sigma l^{2},
\end{aligned}
$$

for

$$
\begin{aligned}
& x \in\left(-\frac{1}{2}, \frac{1}{2}\right) \mid \\
& \left( \pm \frac{1+m}{4}-C|\log l| \frac{\varepsilon}{l}, \pm \frac{1+m}{4}+C|\log l| \frac{\varepsilon}{l}\right) .
\end{aligned}
$$

On the other hand, about the usual Cahn-Hilliard equations we have known the similar and better result to Proposition 3.1. See, for example, Carr, Gurtin and Slemrod ${ }^{10}$ and Alikakos and McKinney. ${ }^{16}$ Combining these, we get the following

Proposition 2.8: Assume that $W$ satisfies $\left(A 1^{\prime}\right),\left(A 2^{\prime}\right)$, $\left(A 3^{\prime}\right),\left(A 4^{\prime}\right)$ and $\left(A 5^{\prime}\right)$, and that $m \in\left(z_{-}, z_{+}\right)$. There exist constants $C$ and $c_{0}$ such that, if

$$
l \leqslant c_{0} \text { and } \varepsilon \leqslant c_{0} \frac{l}{|\log l|},
$$

then

$$
\left|\underline{u}(x)-q_{l}(x)\right| \leqslant C \sigma l^{2},
$$

and

$$
\frac{\varepsilon}{l}\left|\underline{u}_{x}(x)-q_{l}^{\prime}(x)\right| \leqslant C \sigma l^{2},
$$

for $x \in(-1 / 2,1 / 2)$, and, about the $L^{2}$-estimates,

$$
\||\underline{u}|-1\|_{L^{2}}+\frac{\varepsilon}{l}\left\|\underline{u}_{x}\right\|_{L^{2}}+\frac{\varepsilon^{2}}{l^{2}}\left\|\underline{u}_{x x}\right\|_{L^{2}} \leqslant C\left(\frac{\varepsilon}{l}+\sigma l^{2}\right),
$$

and

$$
\left\|W^{\prime}(\underline{u})\right\|_{L^{2}} \leqslant C\left(\frac{\varepsilon}{l}+\sigma l^{2}\right) .
$$

We now state the main estimate in this section. 
Theorem 2.9: Assume that $W$ satisfies $\left(A 1^{\prime}\right),\left(A 2^{\prime}\right)$, $\left(A 3^{\prime}\right),\left(A 4^{\prime}\right)$, and $\left(A 5^{\prime}\right)$, and that $m \in\left(z_{-}, z_{+}\right)$. There exist constants $C$ and $c_{0}$ such that, if

$$
l \leqslant c_{0} \text { and } \varepsilon \leqslant c_{0} \frac{l}{|\log l|},
$$

then there exists a unique global minimizer $\underline{u}$, and $E_{m}(l)$ $=\widetilde{E}_{m}(l)$,

$$
\begin{aligned}
& \left|E_{m}(l)-\left(2 A \varepsilon+\frac{1}{48}\left(1-m^{2}\right)^{2} \sigma l^{3}\right)\right| \leqslant C\left(\frac{\varepsilon}{l}+\sigma l^{2}\right) l^{3}, \\
& \left|E_{m}^{\prime}(l)-\frac{1}{16}\left(1-m^{2}\right)^{2} \sigma l^{2}\right| \leqslant C\left(\frac{\varepsilon}{l}+\sigma l^{2}\right) l^{2} \\
& \left|E_{m}^{\prime \prime}(l)-\frac{1}{8}\left(1-m^{2}\right)^{2} \sigma l\right| \leqslant C\left(\frac{\varepsilon}{l}+\sigma l^{2}\right) l
\end{aligned}
$$

In Ref. 9, we see that the symmetry of $W$ and the fact that $m=0$ are not necessary in the proof of Theorem 5.1 in Sec. V. As is the principally same as of Ref. 9 because of the results of Sec. II and Proposition 3.2, we omit the proof of this theorem in detail in this paper, although we will present another forthcoming paper $^{17}$ to prove the similar estimate for more generalized singular perturbed systems.

\section{PROOF OF THEOREM 1.1}

We first define a normal $n$-layered solution of the EulerLagrange equation of the functional (1.1) in the admissible space $\operatorname{Ad}_{m}$. The Euler-Lagrange equation is the stationary equation of the following time evolution equation:

$$
\begin{aligned}
& u_{t}=-\Delta\left(\varepsilon^{2} \Delta u+f(u)-\sigma\left(-\Delta_{N}\right)^{-1}(u-m)\right), \\
& \quad=-\Delta\left(\varepsilon^{2} \Delta u+f(u)\right)-\sigma(u-m), \quad \text { in } \Omega, \\
& \frac{\partial u}{\partial n}=\frac{\partial(\Delta u)}{\partial n}=0, \quad \text { on } \partial \Omega, \\
& \bar{u}(t)=\frac{1}{|\Omega|} \int_{\Omega} u(x, t) d x=m,
\end{aligned}
$$

with adequate initial data, where $m$ is a given constant, $f(z)=-W^{\prime}(z)$

$$
H_{N, m}^{2}(\Omega)=\left\{u \in H^{2}(\Omega) ; \frac{\partial u}{\partial n}=0, \frac{1}{|\Omega|} \int_{\Omega} u(x) d x=m\right\},
$$

and the definition domain of $A=\Delta^{2}$ is given by

$$
D(A)=\left\{u \in H_{N, m}^{2}(\Omega) ; \Delta u \in H_{N, 0}^{2}(\Omega)\right\} .
$$

We reduce the fourth-order equation to coupled secondorder equations suitable for our method. Let

$$
y=\varepsilon^{2} \Delta u+f(u),
$$

then (3.1) is equivalent to the following coupled system:

$$
\begin{aligned}
& 0=\varepsilon^{2} \Delta u+f(u)-y, \quad \text { in } \Omega, \\
& u_{t}=-\Delta y-\sigma(u-m), \quad \text { in } \Omega, \\
& \bar{u}(t)=m, \\
& \frac{\partial u}{\partial n}=\frac{\partial y}{\partial n}=0, \quad \text { on } \partial \Omega .
\end{aligned}
$$

Note that $\partial y / \partial n=0$ is equivalent to $\partial \Delta u / \partial n=0$, since $f$ is smooth. The associated stationary problem is given by

$$
\begin{aligned}
& \varepsilon^{2} \Delta u+f(u)-y=0, \quad \text { in } \Omega, \\
& \Delta y+\sigma(u-m)=0, \quad \text { in } \Omega, \\
& \bar{u}=m, \\
& \frac{\partial u}{\partial n}=\frac{\partial y}{\partial n}=0, \quad \text { on } \partial \Omega .
\end{aligned}
$$

Apparently, if and only if the third condition of (3.4) holds, the second equation of (3.4) has a solution for $u \in L^{2}(\Omega)$. On the other hand, due to integrating the first equation of (3.4), we see

$$
\int_{\Omega} y(x) d x=\int_{\Omega} f(u(x)) d x
$$

corresponding to the Lagrange multiplier of the EulerLagrange equation. Suppose we have a steady state solution $U^{*}=\left(u^{*}, y^{*}\right)$ of $(3.3)$; the associated linearized eigenvalue problem around $U^{*}$ is

$$
\begin{aligned}
& \varepsilon^{2} \Delta w+f_{u}\left(u^{*}\right) w-z=0, \quad \text { in } \Omega, \\
& \Delta z+(\sigma+\lambda) w=0, \quad \text { in } \Omega, \\
& \bar{w}=0, \\
& \frac{\partial w}{\partial n}=\frac{\partial z}{\partial n}=0 \quad \text { on } \partial \Omega .
\end{aligned}
$$

Again $\bar{w}=0$ is a necessary and sufficient condition under which the second equation of (3.4) has a solution, unless $\sigma$ $+\lambda=0$. It is clear that the same linearized problem as (3.4) can be obtained, by linearizing the original equation as follows:

$$
\begin{aligned}
& \lambda w=-\Delta\left(\varepsilon^{2} \Delta w+f_{u}\left(u^{*}\right) w\right)-\sigma w, \quad \text { in } \Omega, \\
& \bar{w}=0, \\
& \frac{\partial w}{\partial n}=\frac{\partial(\Delta w)}{\partial n}=0, \quad \text { on } \partial \Omega .
\end{aligned}
$$

The appropriate function space $Y_{0}$ for $w$ is

$$
w \in Y_{0}=\left\{w \in H_{N, 0}^{2}(\Omega) ; \quad \Delta w \in H_{N, 0}^{2}(\Omega)\right\} .
$$

It is a standard result for the semilinear equation (3.1) that the linearized stability implies nonlinear stability (see Ref. 11).

Note that the nonlinearity of (3.4) is of the Fitz-Hugh Nagumo type. It is easily seen that the side condition $\bar{u}=m$ is automatically satisfied by integrating the second equation of (3.4), if there exists a solution of the first and the second equation of (3.4). Especially, we focus on a class of layered 
solutions called a normal $n$-layered solution, which can be obtained from a mono-layered solution by the folding up principle (see Ref. 11). We do not repeat the detailed arguments for the existence, and only present the final result.

Theorem 3.1 (the existence of a normal $n$-layered solution): For a given $m \in(-1,1)$ and $\sigma>0$, there exists a positive integer $n_{0}(m, \sigma) m$, and $\varepsilon_{0}(n)$ for $n \geqslant n_{0}(m, \sigma)$ such that, for any integer $n \geqslant n_{0}(m, \sigma)$, a normal $n$-layered solution $u_{n}^{\varepsilon, \sigma, m}$ exists for $0<\varepsilon<\varepsilon_{0}(n)$. Moreover, we have $n_{0}(m, \sigma) \rightarrow \infty$ as $\sigma \rightarrow \infty$ and $\varepsilon_{0}(n) \rightarrow 0$ as $n \rightarrow \infty$.

Remark: About the normal $n$-layered solution, we see that the following property holds: For given $N \in \mathbf{N}, z_{-}<m$ $<z_{+}$and $\sigma>0$, there exists a constant $\varepsilon_{0}$ such that, if 0 $<\varepsilon<\varepsilon_{0}$, then the normal $N$-layered solution is exponentially stable in the sense of (3.6). For the proof of it, we refer to Ref. 11, because it is almost the same argument.

Let $u_{\min }$ be a global minimizer of $F_{\varepsilon, \sigma}$ in $\mathrm{Ad}_{m}$ and a normal $n$-layered solution for some $n \in \mathbf{N}$. We first suppose that $u_{\min }$ is spatially periodic and let the period be $l_{p}$ $\left(=P^{\varepsilon, \sigma, m}\right)$. In the antiperiodic case, we reduce to the periodic case through the same argument of Sec. VII in Ref. 9. In what follows we always assume the hypotheses of Theorem 1.1 .

We define

$$
\begin{aligned}
& E_{0}=\min \left\{N \widetilde{E}_{m}\left(\frac{1}{N}\right) ; N \in \mathbf{N}\right\}, \\
& E_{1}=\min \left\{N E_{n}\left(\frac{1}{N}\right) ; N \in \mathbf{N}\right\} .
\end{aligned}
$$

We prove the next lemma.

Lemma 3.2: $E_{0}$ and $E_{1}$ are both attained, and $E_{0}=E_{1}$. Moreover, if the attaining number is $N_{0}$, there exist $c_{1}>0$ and $c_{2}>0$ such that

$$
c_{1} \varepsilon^{-1 / 3} \leqslant N_{0} \leqslant c_{2} \varepsilon^{-1 / 3} \text {. }
$$

Proof: If $N_{0} \geqslant W(-1+m / 2) / B(-1, m) \varepsilon$ and $N_{0} \geqslant W(1$ $+m / 2) / B(-1, m) \varepsilon$, or if $N_{0} \geqslant W(-1+m / 2) /|B(1, m)| \varepsilon$ and $N_{0} \geqslant W(1+m / 2) /|B(1, m)| \varepsilon$, then by (2.9), $E_{1} \geqslant C_{m, \sigma}$ for some constant $C_{m, \sigma}$. Otherwise, assume $N_{0} \sim \varepsilon^{-1 / 3}$, by (2.7), $E_{0} \leqslant C \varepsilon^{2 / 3}$. Therefore, these are attained. Furthermore, by (2.9) and (2.10), $E_{1} \geqslant C\left(N_{0} \varepsilon+N_{0}^{-2}\right)$. We thus conclude that $c_{1} \varepsilon^{-1 / 3} \leqslant N_{0} \leqslant c_{2} \varepsilon^{-1 / 3}$. Applying Theorem 2.9 with $l$ $=N_{0}^{-1}$, we see $E_{0}=E_{1}$.

The following proposition is principally the same as Proposition 6.1 in Ref. 9. We thus omit the proof. We use the assumption of periodicity of the global minimizer $u_{\min }$.

Proposition 3.3: There exist positive constants $\varepsilon_{0}$ and $c_{1}$ such that, if $\varepsilon \leqslant \varepsilon_{0}$, then

$$
\int_{0}^{l_{p}} \varepsilon^{2}\left(u_{\text {min }}\right)_{x}^{2}+W\left(u_{\text {min }}\right)+\sigma\left|\int_{0}^{x}\left(u_{\text {min }}(t)-m\right) d t\right|^{2} d x \geqslant c_{1} \varepsilon .
$$

Lemma 3.4: $\min \left\{F_{\varepsilon, \sigma}(u) ; u \in \operatorname{Ad}_{m}\right\}=E_{1}$.

Proof: Let $l_{0}$ be $N_{0}^{-1}\left(\sim \varepsilon^{1 / 3}\right)$. Let $\underline{u}_{0}$ denote the unique global minimizer of $I_{l_{0}}$ in $H_{a d m}$. By parallel translation, we make $\underline{u}_{0}$ satisfy the homogeneous Neumann boundary condition at $x=0,1$, and extend $\underline{u}_{0}$ periodically to the whole line. We denote it as the same $\underline{u}_{0}$. We then define

$$
\widetilde{u}(x)=\underline{u}_{0}\left(l_{0}^{-1} x\right) .
$$

We note that $\widetilde{u}(x) \in \operatorname{Ad}_{m}$. In view of Lemma 2.1,

$$
\min \left\{F_{\varepsilon, \sigma}(u) ; u \in \operatorname{Ad}_{m}\right\} \leqslant N_{0} E\left(\frac{1}{N_{0}}\right)=E_{1} .
$$

We prove the inequality of the other direction. We define

$$
E_{2}=\min \left\{l^{-1} E_{m}(l) ; l \in(0,1)\right\} .
$$

Let $\underline{l}$ be the attaining number of $E_{2}$. By the same argument as in the proof of Lemma 3.2, $\underline{l} \sim \varepsilon^{1 / 3}$ and $E_{2} \sim \varepsilon^{2 / 3}$. Define $f_{m}(l)=l^{-1} E_{m}(l)$. In view of $(2.11),(2.12)$ and (2.13), in $l$ $\sim \varepsilon^{1 / 3}$,

$$
f_{m}^{\prime \prime}(l)=\frac{E_{m}^{\prime \prime}(l)}{l}-\frac{2 E_{m}^{\prime}(l)}{l^{2}}+\frac{2 E_{m}(l)}{l^{3}} \geqslant C\left(1-\varepsilon^{2 / 3}\right)>0,
$$

for $\varepsilon$ small enough. Therefore $\underline{l}$ is unique. Moreover,

$$
\left|E_{1}-E_{2}\right| \leqslant C \min \left\{\left|\underline{l}-\frac{1}{N}\right|^{2} ; N \in \mathbf{N}\right\} \leqslant C \varepsilon^{4 / 3} \text {. }
$$

We define

$$
\begin{aligned}
F_{p}= & \int_{0}^{l_{p}} \varepsilon^{2}\left(u_{\text {min }}\right)_{x}^{2}+W\left(u_{\text {min }}\right) \\
& +\sigma\left|\int_{0}^{x}\left(u_{\text {min }}(t)-m\right) d t\right|^{2} d x .
\end{aligned}
$$

By Proposition 3.3 and the assumption that $u_{\text {min }}$ is a normal n-layered solution,

$$
F_{\varepsilon, \sigma}\left(u_{\text {min }}\right)=N_{p} F_{p} \geqslant N_{p} \max \left(E_{m}\left(l_{p}\right), c_{1} \varepsilon\right),
$$

where $N_{p}=l_{p}^{-1}$.

We fix $c_{0}$ for which Theorem 2.9 holds. By (2.10) and the fact that $F_{\varepsilon, \sigma}\left(u_{\text {min }}\right) \leqslant E_{1} \leqslant C \varepsilon^{2 / 3}, l_{p} \leqslant c_{0}$. Similarly, by (2.9), $N_{p} \leqslant C \varepsilon^{-1 / 3}$.

We now prove that

$$
\frac{l_{p}}{\left|\log l_{p}\right|} \geqslant c_{0}^{-1} \varepsilon
$$

by contradiction. Assume that $l_{p} /\left|\log l_{p}\right|<c_{0}^{-1} \varepsilon$, by (2.9), we see

$$
E_{m}\left(l_{p}\right) \geqslant C \frac{l_{p}}{\left|\log l_{p}\right|} .
$$

Therefore, by Proposition 3.3 and

$$
F_{\varepsilon, \sigma}\left(u_{\text {min }}\right) \geqslant \frac{N_{p}}{2}\left(E_{m}\left(l_{p}\right)+c_{1} \varepsilon\right) \geqslant C\left(\frac{1}{\left|\log \frac{1}{N_{p}}\right|}+N_{p} \varepsilon\right) .
$$

As $N_{p} \leqslant C \varepsilon^{-1 / 3}$, we thus see

$$
\left|E_{1}-E_{2}\right| \geqslant\left(\frac{C}{|\log \varepsilon|}-E_{2}\right)+C N_{p} \varepsilon .
$$

The first term of the right hand side is positive and therefore is bigger than $-C \varepsilon^{5 / 3}$. By using (3.7), we see that

$$
C \varepsilon^{4 / 3} \geqslant-C \varepsilon^{5 / 3}+C N_{p} \varepsilon .
$$

Therefore 


$$
N_{p} \leqslant C \varepsilon^{1 / 3},
$$

but this means $N_{p}=0$ for $\varepsilon$ small. This is a contradiction.

As using Theorem 2.9, we see

$$
F_{\varepsilon, \sigma}\left(u_{\min }\right) \geqslant N_{p} F_{p} \geqslant N_{p} E_{m}\left(\frac{1}{N_{p}}\right) \geqslant E_{1} .
$$

This completes the proof.

(The proof of Theorem 1.1.) By Lemma 3.4 and its proof, we see that

$$
E_{0} \geqslant F_{\varepsilon, \sigma}\left(u_{\text {min }}\right) \geqslant N_{p} F_{p} \geqslant N_{p} E_{m}\left(l_{p}\right) \geqslant E_{0} .
$$

By the strict convexity of $E_{m}$ in the range of $l \sim \varepsilon^{1 / 3}$ and the fact that $F_{p} \geqslant E_{m}\left(l_{p}\right), f_{m}\left(1 / N_{p}\right)=N_{p} E_{m}\left(1 / N_{p}\right)=E_{0}$ and $F_{p}$ $=E_{m}\left(1 / N_{p}\right)$. The rescaling periodic unit of $u_{\text {min }}$ is equal to $\underline{u}$ and uniquely determined (up to sign). But because of the discreteness of wave number, there are, at most, two distinct minimizers (up to sign).

In order to estimate the period $1 / N_{p}$, we calculate

$$
\begin{aligned}
0=f_{m}^{\prime}\left(l_{0}\right) & =l_{0}^{-1} E_{m}^{\prime}\left(l_{0}\right)-l_{0}^{-2} E_{m}\left(l_{0}\right) \\
& =-\frac{2 A \varepsilon}{l_{0}^{2}}+\frac{1}{24}\left(1-m^{2}\right)^{2} \sigma l_{0}+O(\varepsilon),
\end{aligned}
$$

where we have used (2.11), (2.12) and the fact that $l_{0}$ $\sim \varepsilon^{1 / 3}$. Because of the strict convexity,

$$
l_{0}=2\left(\frac{6 A \varepsilon}{\left(1-m^{2}\right)^{2} \sigma}\right)^{1 / 3}+O\left(\varepsilon^{2 / 3}\right) \text {. }
$$

As we use the strict convexity again, if $N_{p}=\left[1 / l_{0}\right]$ (the largest integer $\leqslant 1 / l_{0}$ ) or if $N_{p}=\left[1 / l_{0}\right]+1$, then

$$
\frac{1}{N_{p}}=2\left(\frac{6 A \varepsilon}{\left(1-m^{2}\right)^{2} \sigma}\right)^{1 / 3}+O\left(\varepsilon^{2 / 3}\right) \text {. }
$$

For the estimate of energy, we see

$$
E_{0}=f\left(l_{0}\right)+O\left(\varepsilon^{4 / 3}\right)=\frac{\sigma}{4}\left(6\left(1-m^{2}\right) A \frac{\varepsilon}{\sigma}\right)^{2 / 3}+O\left(\varepsilon^{4 / 3}\right) .
$$

This completes the proof.

\section{STABILITY ANALYSIS OF SPATIALLY HOMOGENEOUS STATE AND NUMERICAL EXPERIMENTS}

First of all, we assume (A1), (A2), (A3), (A4), $\Omega$ $=(0,1)$, and $m \in(-1,1)$ in this section. We may also assume that $m^{\prime}=\alpha_{0}=\beta_{0}=0$. We would like to see the whole evolution of the system, although this is more complicated. In the first step, we study the linear stability of the trivial (spatially homogeneous) stationary solution $(u \equiv m)$ of the equation (3.1). Namely, we calculate the eigenvalue $\lambda$ of (3.6), when $u^{*} \equiv m$. For the purpose of comparison, we also consider the following evolution equation:

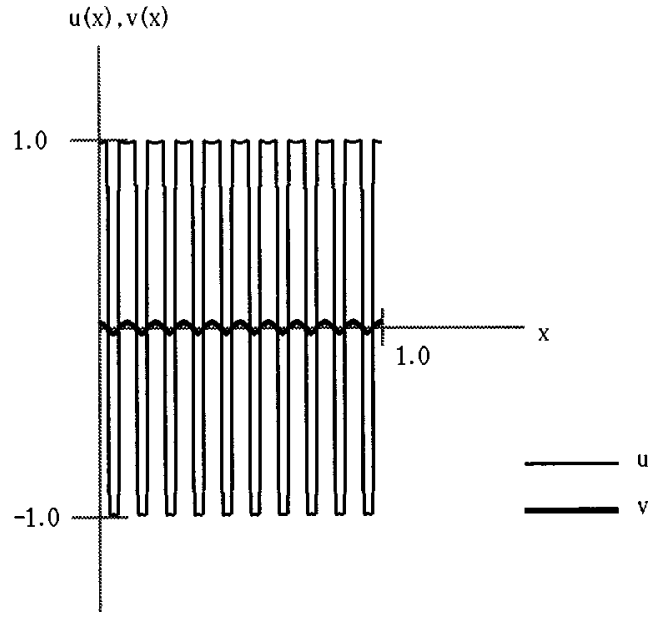

FIG. 2. Shape of the global minimizer as $m=0.25$.

$$
\begin{aligned}
& u_{t}=\varepsilon^{2} u_{x x}+f(u)-\sigma\left(-\Delta_{N}\right)^{-1}(u-m)+\mu, \quad \text { in }(0,1), \\
& u_{x}(0)=u_{x}(1)=0, \\
& \bar{u}(t)=m \\
& u(x, 0)=u_{0}(x),
\end{aligned}
$$

where the notation is the same as in Sec. III, and $\mu$ is equal to $-\int_{0}^{1} f(u(x)) d x$ and is the constant dependent globally upon $u$. Because of $\mu,(4.1)$ is a mass conserved system, like (3.1). The corresponding second-order eigenvalue problem is the following:

$$
\begin{aligned}
& \theta \phi=\varepsilon^{2} \phi_{x x}+f_{u}\left(u^{*}\right) \phi-\sigma\left(-\Delta_{N}\right)^{-1} \phi, \quad \text { in }(0,1), \\
& \phi x(0)=\phi x(1)=0, \\
& \bar{\phi}=0 .
\end{aligned}
$$

The equation (3.1) is called the nonlocal Cahn-Hilliard equation and is the gradient flow of the functional (1.1) in the admissible space $\mathrm{Ad}_{m}$ under $H^{-1}$-topology (refer to Ref. 18 and the references therein), which is a fourth-order parabolic partial differential equation describing the dynamics of the system. On the other hand, the second-order parabolic

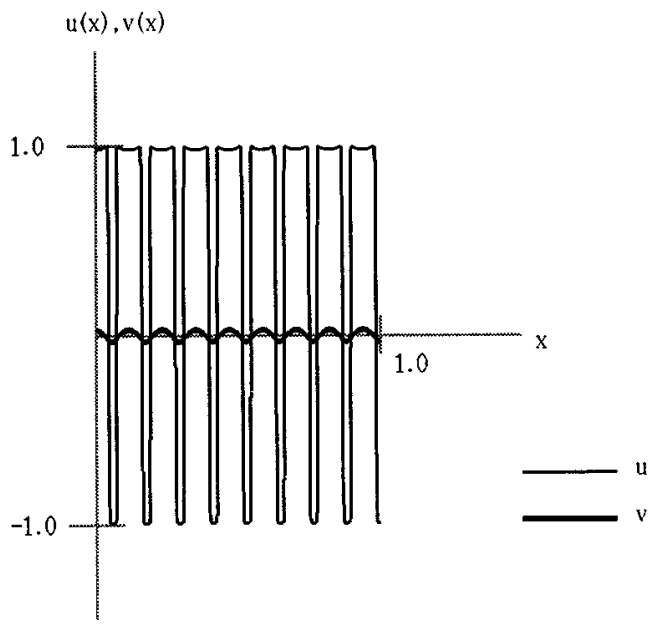

FIG. 3. Shape of the global minimizer as $m=0.5$. 


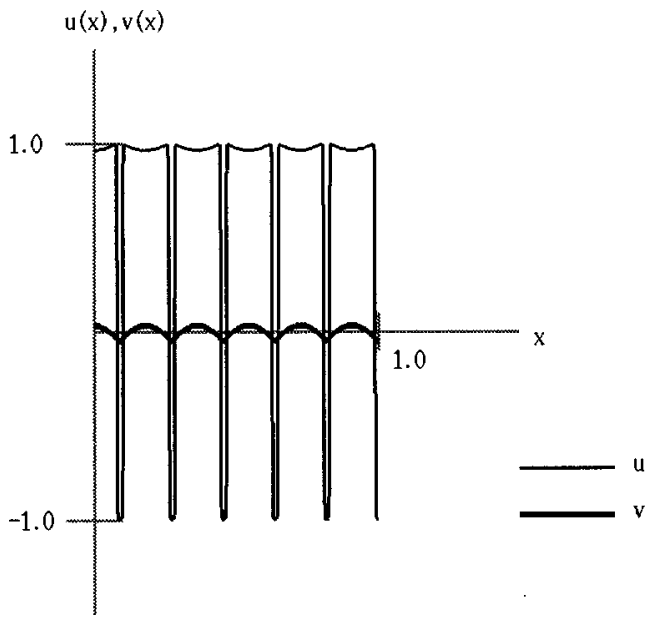

FIG. 4. Shape of the global minimizer as $m=0.75$.

partial differential equation (4.1) with nonlocality is the gradient flow of the same functional (1.1) in the same admissible space $\operatorname{Ad}_{m}$ under $L^{2}$-topology. As is shown in Ref. 18, both gradient flows do not have only the same stationary states, but also their linear stabilities of an arbitrary stationary solution agree with each other. Therefore, if we are interested only in the linear stability of the stationary states, we can adopt both. But, of course, the evolutions have some difference between the second and the fourth time dependent equation.

We now consider $u^{*} \equiv m$ here, which is, of course, a stationary solution of both (3.1) and (4.1). We put $e^{i k x}$ into $w$ in (3.6) and compute the dispersion relation, i.e., the dependency of $\lambda$ upon wavenumber $k \geqslant 0$ :

$$
\begin{aligned}
& \lambda(k)=-\varepsilon^{2} k^{4}+f^{\prime}(m) k^{2}-\sigma, \\
& \frac{d \lambda}{d k}(k)=2 k\left(-2 \varepsilon^{2} k^{2}+f^{\prime}(m)\right) .
\end{aligned}
$$

Because of (4.4), if $f^{\prime}(m) \leqslant 0, \lambda(k)<0$ for any $k \geqslant 0$, and the maximum value of $\lambda(k)$ equals $-\sigma$, when $k=0$. Hence, $u$ $\equiv m$ is linearly stable. On the other hand, if $f^{\prime}(m)>0$, for any given $\sigma>0$, there exists $\varepsilon_{2}>0$ such that $\lambda(k)$ has the positive maximum $-\sigma+f^{\prime}(m)^{2} / 4 \varepsilon^{4}$ for $0<\varepsilon<\varepsilon_{2} . u \equiv m$ is

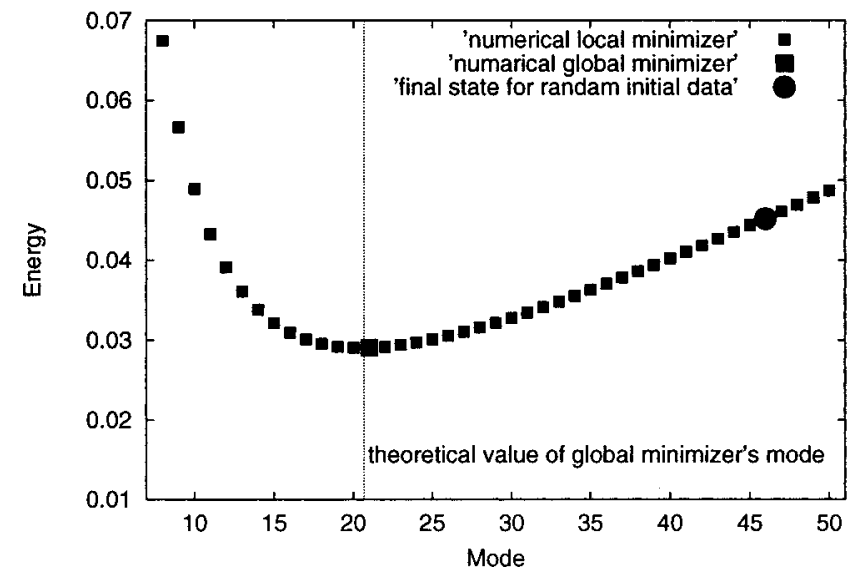

FIG. 5. The mode-energy graph of stable stationary states as $m=0.0$.

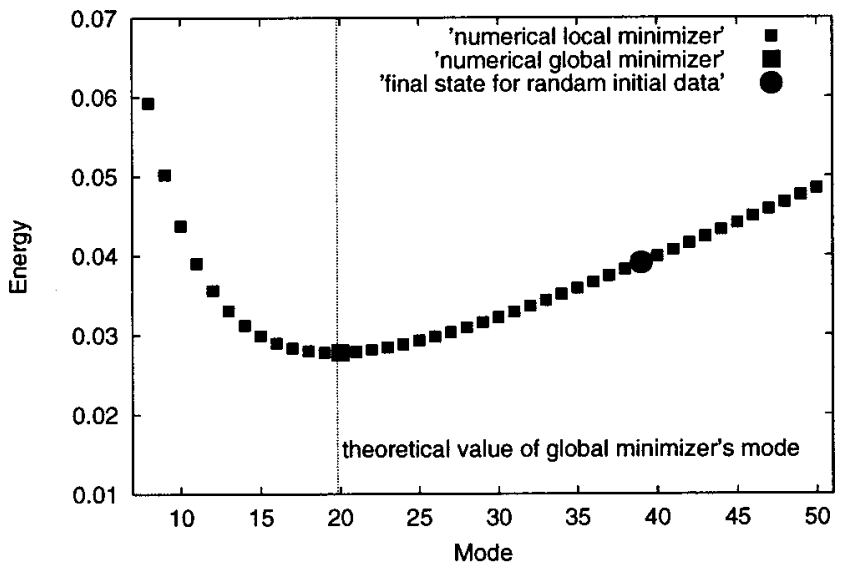

FIG. 6. The mode-energy graph of stable stationary states as $m=0.25$.

therefore linearly unstable, and the most unstable wavelength is in $O(\varepsilon)$ for fixed $f^{\prime}(m)$ and $\sigma$.

For the purpose of comparison, we also compute $\theta$ in (4.2) similarly

$$
\begin{aligned}
& \theta(k)=-\varepsilon^{2} k^{2}+f^{\prime}(m)-\frac{\sigma}{k^{2}}, \\
& \frac{d \theta}{d k}(k)=-2 k^{-3}\left(\varepsilon^{2} k^{4}-\sigma\right) .
\end{aligned}
$$

Hence, the maximum eigenvalue is $f^{\prime}(m)-2 \varepsilon \sqrt{\sigma}$, when $k$ $=\varepsilon^{-1 / 2} \sigma^{1 / 4}$. We see, if $f^{\prime}(m) \leqslant 0$, that $u \equiv m$ is linearly stable, and otherwise, linearly unstable. Moreover, the most unstable wavelength is in $O(\sqrt{\varepsilon})$ for fixed $f^{\prime}(m)$ and $\sigma$. We note that $f^{\prime}(m)>0$ corresponds to $m \in\left(z_{-}, z_{+}\right)$, called the "spinodal region," and $f^{\prime}(m)<0$ to $m \in$ $\left(-1, z_{-}\right) \cup\left(z_{+}, 1\right)$, called the "metastable region." We also add that, in both cases, all the unstable wavelengths are in $O\left(\varepsilon^{\delta}\right)(1 \geqslant \delta \geqslant 1 / 4)$. (The agreement with each other case follows also from the general theory of Ref. 18.) We conclude that, if $m$ is in the spinodal region and if the homogeneous steady state $\left(u^{*} \equiv m\right)$ gets instability, the most rapidly unstable mode in both cases (3.1) and (4.1) is different from the global minimizers' wavelength $O\left(\varepsilon^{1 / 3}\right)$ derived from Theorem 1.1. That is finer than this, because of $1>1 / 2$

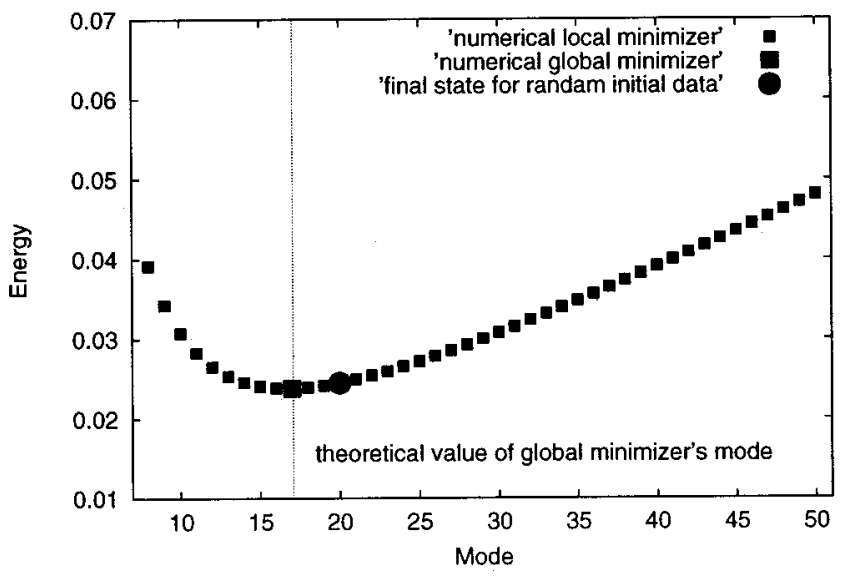

FIG. 7. The mode-energy graph of stable stationary states as $m=0.50$. 


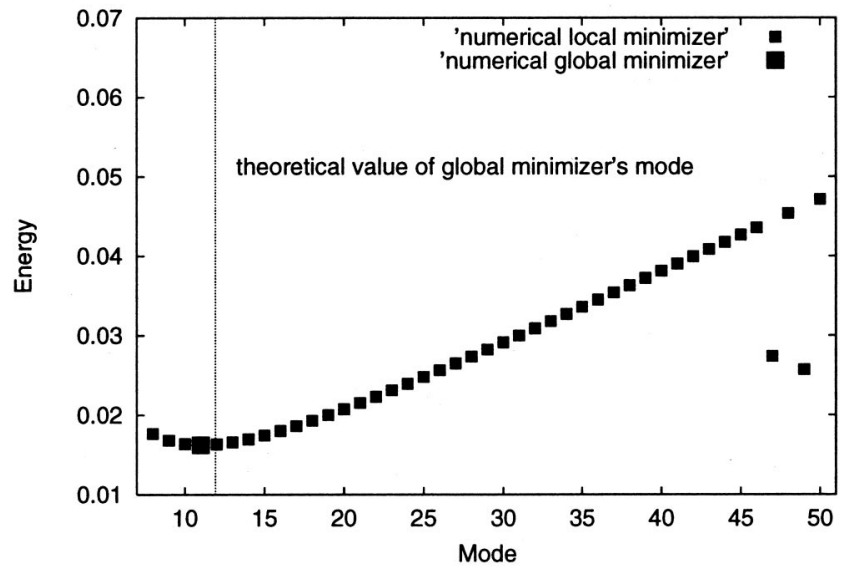

FIG. 8. The mode-energy graph of stable stationary states as $m=0.75$.

$>1 / 3$. We can regard it as a reason why spinodal decomposition first occurs and the coarsening process next occurs. But, as we see from Theorem 3.1 and its remark, if $m$ is in spinodal region, there are many exponentially stable stationary solutions of (3.1) for $\varepsilon$ small enough. The solution starting from the homogeneous unstable state may or may not reach a global minimizer (strong segregation limit) of the free energy functional (1.1) via the coarsening process. The evolution has some kind of sensitivity about the initial data. In some of the following paragraphs, we investigate more about it by numerical experiments, although we do not know any tools to study rigorously a weak segregation limit. Here we define a weak segregation limit as the final state starting from the trivial steady state $u \equiv m$ perturbed randomly.

In the remaining part of this section we present some numerical experiments for reinforcement of our theoretical results, for a suggestion to new results, and for understanding the dynamics of the system more. We first explain our method. We adopt a finite difference method for discretization. The spatial mesh size is $1 / 10000$ (the number of total spatial points are 10001 including two boundary points). The double-well potential, $W(u)$, is defined as $1 / 4\left(u^{2}-1\right)^{2}$. We note that $m^{\prime}=\alpha_{0}=\beta_{0}=0$ and $z_{ \pm}= \pm 1 / \sqrt{3}$ in this case.

We adopt the discretization of the gradient flow (4.1) in order to get the stationary states. Generally speaking, the parameter domain of the second-order parabolic partial differential equation in which numerical computation is stable and succeeded is wider than of the fourth-order parabolic one. It is true in our problem. This is why we use the secondorder one. But, only when we calculate weak segregation limits, we use the discretization of (3.1).

The condition for judging convergence is that the sum of the square of all differences at every spatial point between the $n$-th, and the $n+1$-th calculation is less than 1.0 $\times 10^{-10}$.

When $\varepsilon=0.001, \sigma=100$, the shapes of the global minimizers $u$ of $F_{\varepsilon, \sigma}$ and the corresponding $v$ vary as Figs. 1, 2, 3 and 4, accordingly, as $m$ changes from 0.0 to 0.75 (refer also to the Euler-Lagrange equation of $F_{\varepsilon, \sigma}$ in $\mathrm{Ad}_{m}$ ). These are all spatially periodic or antiperiodic and are normal $n$-layered solutions for some $n \in \mathbf{N}$.

Figures 5, 6, 7 and 8 are mode-energy graphs of stable stationary states, when $\varepsilon=0.001$ and $\sigma=100$. An $x$-axis rep-
TABLE I. A comparison between numerical and theoretical values of the wavelength of global minimizers.

\begin{tabular}{|c|c|c|c|c|}
\hline$\varepsilon=0.010$ & $\sigma=50$ & 80 & 100 & 150 \\
\hline \multirow[t]{3}{*}{$m=0.0$} & mode 7 & mode 9 & mode 9 & mode 10 \\
\hline & 0.285713 & 0.222221 & 0.222221 & 0.200014 \\
\hline & $(0.262568)$ & $(0.224492)$ & $(0.208400)$ & $(0.182054)$ \\
\hline \multirow[t]{3}{*}{0.25} & mode 7 & mode 8 & mode 9 & mode 10 \\
\hline & 0.285709 & 0.250013 & 0.222217 & 0.200010 \\
\hline & $(0.274112)$ & $(0.234362)$ & $(0.217563)$ & $(0.190058)$ \\
\hline \multirow[t]{3}{*}{0.5} & mode 6 & mode 7 & mode 7 & mode 8 \\
\hline & 0.333347 & 0.285704 & 0.285703 & 0.250009 \\
\hline & $(0.318078)$ & $(0.271953)$ & $(0.252459)$ & $(0.220543)$ \\
\hline \multirow[t]{3}{*}{0.6} & mode 5 & mode 5 & mode 6 & Constant \\
\hline & 0.399964 & 0.399954 & 0.333346 & Local Min \\
\hline & $(0.353553)$ & $(0.302284)$ & $(0.280616)$ & $(0.245140)$ \\
\hline$\varepsilon=0.005$ & $\sigma=50$ & 80 & 100 & 150 \\
\hline \multirow[t]{3}{*}{$m=0.0$} & mode 9 & mode 11 & mode 12 & mode 13 \\
\hline & 0.222226 & 0.181821 & 0.166675 & 0.153845 \\
\hline & $(0.208400)$ & $(0.178180)$ & $(0.165407)$ & $(0.144497)$ \\
\hline \multirow[t]{3}{*}{0.25} & mode 9 & mode 10 & mode 11 & mode 13 \\
\hline & 0.222219 & 0.200009 & 0.181816 & 0.153844 \\
\hline & $(0.217563)$ & $(0.186013)$ & $(0.172680)$ & $(0.150849)$ \\
\hline \multirow[t]{3}{*}{0.5} & mode 8 & mode 9 & mode 9 & mode 10 \\
\hline & 0.250009 & 0.222218 & 0.222217 & 0.200007 \\
\hline & $(0.252459)$ & $(0.215849)$ & $(0.200377)$ & $(0.175045)$ \\
\hline \multirow[t]{3}{*}{0.6} & mode 7 & mode 8 & mode 8 & mode 9 \\
\hline & 0.285707 & 0.250008 & 0.250007 & 0.222215 \\
\hline & $(0.280616)$ & $(0.239923)$ & $(0.222725)$ & $(0.194568)$ \\
\hline$\varepsilon=0.001$ & $\sigma=50$ & 80 & 100 & 150 \\
\hline \multirow[t]{3}{*}{$m=0.0$} & mode 16 & mode 19 & mode 21 & mode 23 \\
\hline & 0.125004 & 0.105263 & 0.095238 & 0.086956 \\
\hline & $(0.121873)$ & $(0.104200)$ & $(0.096731)$ & $(0.084502)$ \\
\hline \multirow[t]{3}{*}{0.25} & mode 16 & mode 18 & mode 20 & mode 22 \\
\hline & 0.125004 & 0.111115 & 0.100003 & 0.090912 \\
\hline & $(0.127231)$ & $(0.108781)$ & $(0.100984)$ & $(0.088217)$ \\
\hline \multirow[t]{3}{*}{0.5} & mode 13 & mode 16 & mode 17 & mode 19 \\
\hline & 0.153844 & 0.125003 & 0.117645 & 0.105262 \\
\hline & $(0.147639)$ & $(0.126229)$ & $(0.117181)$ & $(0.102367)$ \\
\hline \multirow[t]{3}{*}{0.75} & mode 9 & mode 10 & mode 11 & mode 13 \\
\hline & 0.222219 & 0.200004 & 0.181815 & 0.153843 \\
\hline & $(0.211474)$ & $(0.180807)$ & $(0.167847)$ & $(0.146628)$ \\
\hline
\end{tabular}

resents the mode of stationary states and the $y$-axis is energy. If the initial data is made, the type of $A_{n} \cos n \pi x\left(A_{n}\right.$ is the amplitude and $n$ means the mode) and we solve (4.1) numerically, the same mode stable steady state is obtained. Theoretically, Theorem 3.1 tells us about the multiple existence of stable stationary states, only when $\varepsilon$ is very small. But the numerical computations means that this fact holds in more parameter values. According to figures, numerical global minimizers agree with theoretical ones very much, even if $m$ is out of the spinodal region.

The weak segregation limit is indicated by the black small disk in the figures. If $m$ is in the spinodal region, then the wavelength is finer than the one of the global minimizer. The smaller the difference is, the bigger $m$ is. On the other hand, if $m$ is in the metastable region (in the case of Fig. 8), the solution starting from $u \equiv m$ perturbed randomly con- 


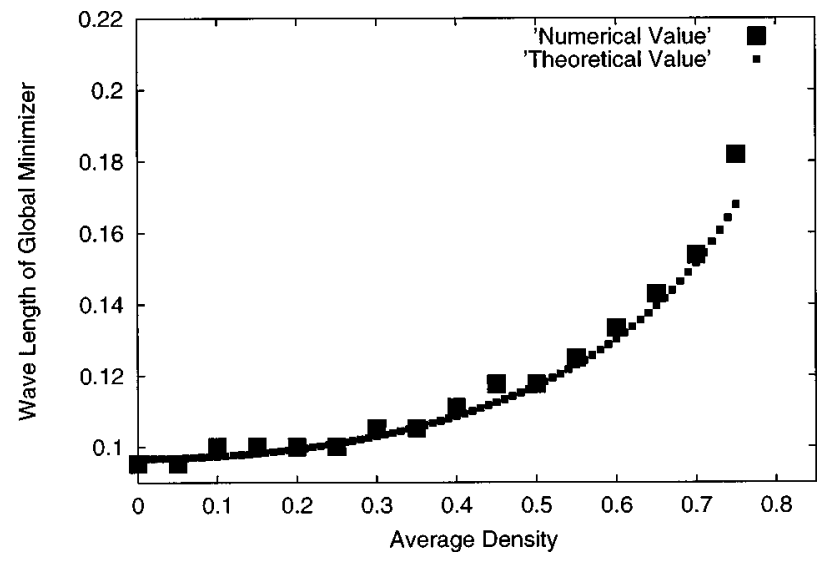

FIG. 9. The $m$-wavelength graph as $\varepsilon=0.001$ and $\sigma=100$.

verges to the same trivial solution $u \equiv m$, when the perturbation is rather bigger. In this case, we make sure the linear stability of $u \equiv m$ numerically. We see a longer wavelength (and rather bigger) perturbation needed in order not to converge to $u \equiv m$.

See Table I. These are the comparisons of the theoretical values with the numerical ones of the wavelength, $P^{\varepsilon, \sigma, m}$, of the global minimizers of $F_{\varepsilon, \sigma}$ in $\mathrm{Ad}_{m}$ in more parameter values. The numbers in the bracket $(\cdot)$ in the table mean the theoretical values given by (1.4). Theorem 1.1 is obtained only in very small $\varepsilon$, although (1.4) agrees with the numerical result in a broad range of parameters in our numerics. Especially note that 0.6 and $0.75>1 / \sqrt{3}\left(=z_{+}\right)$. This suggests that the assumption about $m$ is technical and may not be necessary. When $\varepsilon=0.001$ and $\sigma=100$, we compute it in much more average density values and make the $m$-wavelength graph in Fig. 9. We consider that the tendency of changing wavelength agrees with each other.

\section{ACKNOWLEDGMENTS}

We thank Professor Motohiko Yamaguchi in the Agency of Industrial Science and Technology M.I.T.I. for his inform- ing us of the special issue of Chaos and for his introducing the first author to the fourth. We also wish to thank Professor John A. Pojman for the careful proofreading and many helpful comments.

${ }^{1}$ D. Henry, Geometric Theory of Semilinear Parabolic Equations (Lecture Note in Mathematics 1st ed. Springer-Verlag, Berlin, 1981), p. 840.

${ }^{2} \mathrm{~T}$. Ohta and K. Kawasaki, "Equilibrium morphology of block copolymer melts," Macromolecules 19, 2621-2632 (1986).

${ }^{3}$ M. Bahiana and Y. Oono, Phys. Rev. 41, 6763 (1990).

${ }^{4}$ Y. Nishiura and I. Ohnishi, "Some mathematical aspects of the microphase separation in diblock copolymers,"' Physica D 84, 31-39 (1995).

${ }^{5}$ H. Hasegawa, H. Tanaka, K. Yamasaki, and T. Hashimoto, Macromolecules 20, 1651 (1987).

${ }^{6}$ T. Hashimoto, M. Shibayama, and H. Kawai, Macromolecules 16, 1093 (1983).

${ }^{7}$ T. Hashimoto, H. Tanaka, and H. Hasegawa, Molecular Conformation and Dynamics of Macromolecules in Condensed Systems, edited by M. Nagasawa (Elsevier, Amsterdam, 1998)

${ }^{8}$ Y. Matsushita, K. Mori, R. Saguchi, Y. Nakao, I. Noda, and M. Nagasawa, Macromolecules 23, 4313-4316 (1990).

${ }^{9}$ S. Müller, "Singular perturbations as a selection criterion for periodic minimizing sequences," Calc. Var. 1, 169-204 (1993).

${ }^{10}$ J. Carr, M. E. Gurtin, and M. Slemrod, "Structured phase transitions on a finite interval," Arch. Ration. Mech. Anal. 86, 317-351 (1984).

${ }^{11}$ Y. Nishiura, Dynamics Reported (New Series) (Springer-Verlag, Berlin, 1994), Vol. 3.

${ }^{12} \mathrm{P}$. Fife, "Boundary and interior transition layer phenomena for pairs of second order differential equations," J. Math. Anal. Appl. 54, 497-521 (1976).

${ }^{13}$ M. Mimura, M. Tabata, and Y. Hosono, "Multiple solutions of two-point boundary value problems of Neumann type with a small parameter," SIAM J. Math. Anal. 11, 613-631 (1980).

${ }^{14}$ M. Ito, "A remark on singular perturbation methods," Hiroshima Math. J. 14, 619-629 (1985).

${ }^{15}$ Y. Nishiura and H. Fujii, "Stability of singularly perturbed solutions to systems of reaction diffusion equations," SIAM (Soc. Ind. Appl. Math.) J. Math. Anal. 18, 1726-1770 (1987).

${ }^{16}$ N. D. Alikakos and W. R. McKinney, "Remarks on the equilibrium theory for the Cahn-Hilliard equation in one space dimension,"' ReactionDiffusion Equations (Oxford University Press, Oxford, 1990), pp. 75-93.

${ }^{17}$ I. Ohnishi and Y. Nishiura, in preparation.

${ }^{18}$ I. Ohnishi and Y. Nishiura, "Spectral comparison between the second order and fourth order equations of conservative type with non-local terms,'’ Jpn. J. Ind. Appl. Math. 15, 253-262 (1998). 